\title{
Influenza and pneumococcal vaccination: patient perceptions
}

\author{
Paul F Findlay, Y M Gibbons, W R Primrose, G Ellis, G Downie
}

\begin{abstract}
The efficacy of the influenza vaccine in reducing mortality and hospital admissions is established, particularly in the elderly. However, up to $50 \%$ of those at risk do not receive the vaccine. These patients are also at risk from pneumococcal infection and there is considerable overlap between the target group for each vaccine.

This study sought to identify at risk individuals from consecutive admissions to an acute geriatric unit and to gain an insight into their perceptions with regard to vaccination. The awareness of each vaccine was recorded, together with the vaccination history.

Seventy four per cent of the final cohort had heard of the influenza vaccine, while only $13 \%$ had heard of the pneumococcal vaccine. Fifty per cent perceived themselves to be at risk from influenza and its complications and $87 \%$ of the cohort believed it to be a serious infection.

Influenza vaccine was judged to confer good protection by $72 \%$ of the sample and yet up to $50 \%$ believed that the vaccine can make the recipient ill.

Influenza is perceived as a serious infection by patients and yet many do not believe themselves to be at particular risk. Although influenza vaccination is believed to confer protection, the decision whether, or not, to accept the vaccine is coloured by many factors, including popular myths and anecdotal information from friends and relatives. The uptake of influenza vaccine is suboptimal and the awareness of the pneumococcal vaccine certainly in the elderly is poor. The need for a comprehensive nationwide education campaign promoting both influenza and pneumococcal vaccine is highlighted.

(Postgrad Med f 2000;76:215-217)
\end{abstract}

Keywords: influenza vaccine; pneumococcal vaccine

Department of Medicine for the Elderly, Woodend Hospital, Eday Road, Aberdeen AB15 6LS, UK

P F Findlay

Y M Gibbons

W R Primrose

G Ellis

G Downie

Correspondence to: Dr Findlay

Submitted 1 March 1999 Accepted 6 September 1999 Influenza is a common infectious disease that can affect up to $20 \%$ of the population each year and in the elderly it is a major cause of morbidity and mortality. The most recent UK epidemic resulted in more than 20000 deaths, the majority in people over 65 years old. ${ }^{1}$ The Department of Health has recently extended the recommendations for influenza vaccination to include individuals aged 75 or over. Although the vaccines are poor at preventing the primary infection, they reduce the incidence of bronchopneumonia, hospital admissions, and mortality in vulnerable groups. ${ }^{2}$ As
Box 1: Influenza vaccination indications (Department of Health)

- Chronic respiratory disease

- Chronic heart disease

- Chronic renal disease

- Diabetes mellitus and other endocrine disorders

- Immunosuppressed patients

- Residents of nursing homes and other long stay wards

- All patients aged 75 or over

Side effects

- Local erythema

- Mild systemic illness

- Demyelination syndrome

Contraindications

- Allergy to eggs

- Previous adverse reaction

the complications of influenza are more common in the elderly this group derives greatest benefit from vaccination.

The target population for influenza vaccination as defined by the Department of Health (box 1) overlaps considerably with those deemed to be at risk from pneumococcal infection (box 2). Streptococcus pneumoniae is the most common aetiological agent in community acquired pneumonia, accounting for over $60 \%$ of cases. ${ }^{3}$ Pneumococcal vaccine is active against 23 capsule types including the commonest serotypes, which account for more than $90 \%$ of infections in adults and $85 \%$ in children. ${ }^{45}$ The overall efficacy of the vaccine is estimated to be between $60 \%-70 \%{ }^{6}$ The vaccine is, however, seriously underused and the uptake of pneumococcal in comparison to that of the influenza vaccine is very poor. Indeed it is estimated that only $10 \%$ of those at risk actually receive the pneumococcal vaccine..$^{5-7}$

Recent reports have appeared in the litera-

endorsing pneumococcal vaccine along with influenza vaccine and the infrastructure is already in place in a general practice setting to improve the uptake of this vaccine. Unlike the influenza vaccine pneumococcal vaccine is given every five years and those at risk of influenza infection fall into the target group for pneumococcal vaccination. It would therefore be convenient to administer the vaccine while carrying out influenza vaccination.

The aim of this study was to identify at risk individuals from consecutive admissions to an 


\author{
Box 2: Pneumococcal vaccination \\ indications (Department of Health) \\ - Chronic respiratory disease \\ - Chronic heart disease \\ - Chronic renal disease \\ - Diabetes mellitus \\ - Immunodeficiency states \\ - Hyposplenism \\ - Chronic liver disease (including \\ cirrhosis) \\ Side effects \\ - Erythema, induration, and pain $(50 \%)$ \\ - Low grade pyrexia (1\%) \\ - Anaphylaxis (rare) \\ Contraindications \\ - Age less than 2 years \\ - Pregnancy \\ - Breast feeding \\ - Acute infection \\ - Pneumococcal vaccination in the last \\ three years
}

acute geriatric unit and to gain an insight into patient perceptions and general awareness with reference to vaccination.

\begin{tabular}{l} 
Table 1 Questionnaire \\
Part 1 \\
Admission date \\
Date screened \\
Date of birth \\
Sex \\
Abbreviated mental test \\
1. Age \\
2. Time (nearest hour) \\
3. Address (42 West Street) \\
4. What year is it? \\
5. Name of institution \\
6. Recognition of two persons \\
7. Date of birth \\
8. Year of World War 1 \\
9. Name of present monarch \\
10. Count backwards from 20 to 1 \\
Part 2 \\
If the mental test score was greater than or equal to 7 the \\
remainder of the questionnaire continued: \\
With regard to the influenza and pneumococcal vaccines: \\
1. Have you heard of either vaccine? \\
2. Has vaccination been offered to you with either or \\
both? \\
3. Would you accept either or both? \\
4. Have you had vaccination with the either vaccine? \\
Do you agree or disagree with the following: \\
5. My general health is good \\
6. I am at risk from influenza \\
7. Complications of influenza are serious \\
8. Influenza vaccination gives good protection \\
9. Influenza vaccination can make you ill \\
Disease category (may be more than one in each patient) \\
Chronic respiratory \\
Chronic cardiac disease \\
Chronic renal disease \\
Diabetes mellitus \\
Hyposplenism \\
Immunodeficiency (including steroid therapy) \\
Chronic liver disease (if only indication for pneumococcal \\
vaccine only) \\
Exclusion criteria \\
Previous adverse reaction to either vaccine \\
Allergy to eggs (contraindicates administration of \\
Pneumocination within last 5 years \\
\hline
\end{tabular}

Table 2 Results

\begin{tabular}{|c|c|c|c|}
\hline & Yes & No & Unsure \\
\hline \multicolumn{4}{|c|}{ Have you heard of the following vaccines } \\
\hline Influenza & 34 & 2 & 10 \\
\hline Pneumococcal & 6 & 30 & 10 \\
\hline \multicolumn{4}{|c|}{ Has vaccination been offered to you? } \\
\hline Influenza & 25 & 18 & 3 \\
\hline Pneumococcal & 1 & 42 & 3 \\
\hline \multicolumn{4}{|l|}{ Would you accept? } \\
\hline Influenza vaccine & 28 & 12 & 6 \\
\hline Pneumococcal vaccine & 22 & 17 & 7 \\
\hline \multicolumn{4}{|c|}{ Have you had vaccination with the following vaccines? } \\
\hline Influenza & 21 & 21 & 4 \\
\hline Pneumococcal & 2 & 2 & 42 \\
\hline \multicolumn{4}{|l|}{ Is your own health? } \\
\hline Good & 19 & & \\
\hline Not good & 25 & & \\
\hline Unknown & 2 & & \\
\hline \multicolumn{4}{|l|}{ I am at risk from influenza } \\
\hline Agree & 23 & & \\
\hline Disagree & 18 & & \\
\hline Unknown & 5 & & \\
\hline \multicolumn{4}{|c|}{ Complications of influenza are serious } \\
\hline Agree & 40 & & \\
\hline Disagree & 2 & & \\
\hline Unknown & 4 & & \\
\hline \multicolumn{4}{|c|}{ Influenza vaccination gives good protection } \\
\hline Agree & 33 & & \\
\hline Disagree & 2 & & \\
\hline Unknown & 11 & & \\
\hline \multicolumn{4}{|c|}{ Influenza vaccination can make you ill } \\
\hline Agree & 21 & & \\
\hline Disagree & 19 & & \\
\hline Unknown & 6 & & \\
\hline
\end{tabular}

\section{Method}

A research nurse interviewed and completed a simple questionnaire for each patient (table 1). The individual's general awareness of each vaccine was recorded, together with their vaccination history. A total of 119 patients were screened during the two month study period and at risk groups established. Of these, after excluding those with a mental test score of less than 7 , taken to indicate cognitive impairment, and those who did not wish to participate in the study, a final cohort of 46 patients were recruited. Some patients did not complete every question, so the number of responses for each question varies between 36 and 46 .

\section{Results}

The results are shown in table 2 .

\section{Discussion}

Our study illustrates some of the problems and barriers to an effective vaccination programme. It is clear that influenza uptake remains suboptimal and we must therefore look at the reasons why this should be so. An awareness of the vaccine is fundamental and the patient must agree to accept the vaccine if offered. So often this represents a barrier. As we have demonstrated in this small study, awareness of pneumococcal vaccine, for example, is poor and hence the demand for this vaccine is low as the general population is largely unaware of its existence. Even with the influenza vaccine 18 patients, all of whom all fell within a defined high risk group, were not offered the vaccine. Research supports the suggestion that the general practitioner can have an enormous impact on vaccine uptake by recommending the vaccine in the first place. Commitment to a vaccination programme by health care workers would have a major impact on vaccine uptake. 
In addition anecdotal stories of previous bad experiences with regard to, for example, influenza vaccine will certainly affect the uptake. In our study this was clearly demonstrated.

This was perhaps surprising as many of the population sample correctly considered themselves to be at risk from influenza infection and its sequelae. Twenty three of the patients considered themselves to be at risk from influenza infection, while 18 did not. The complications of influenza were recognised as being serious by 40 of the total sample and 33 believed that influenza vaccine conferred good protection. The subjective assessment of general health revealed that 19 considered themselves to enjoy good health, while 25 stated that their health was not good. Yet despite this the decision whether or not to have the influenza vaccine was significantly influenced by anecdotal stories and popular myths from friends and relatives. This clearly underlines the need for a concerted campaign to disseminate information and dispel false beliefs. Such an approach has the potential to yield major benefits and would enhance vaccine uptake.

It is possible to increase the uptake of pneumococcal vaccine with a concerted campaign in a general practice setting as demonstrated in Tameside Family Health Services Authority, which involved 37 practices. Despite the fact that patients' prior awareness of the vaccine was low the proportion of at-risk patients vaccinated increased ninefold after the launch of the campaign. ${ }^{8}$

At present, however, there is little incentive for general practitioners to offer the pneumococcal vaccine as no item of service fee is available, unlike the situation with regard to influenza vaccine. As the vaccine costs approxi- mately $£ 10$ per vial, purchasing a large number of units, without reimbursement, would clearly have an adverse affect on an individual practice's cash flow. Clearly an effective vaccination campaign requires a release of adequate funding to general practitioners. In conclusion, influenza is perceived as a serious infection by patients and yet many do not believe themselves to be at particular risk. Although influenza vaccination is believed to confer protection, the decision whether, or not, to accept the vaccine is coloured by many factors, including popular myths and anecdotal information from friends and relatives. The uptake of influenza vaccine is suboptimal and the awareness of the pneumococcal vaccine is poor. The need for a comprehensive nationwide education campaign promoting both influenza and pneumococcal vaccine is highlighted together with the requirement for the release of adequate funding, to primary care physicians, to ensure that at risk individuals are being appropriately treated.

1 Ashley J, Smith T, Dunnell K. Deaths in Great Britain associated with the influenza epidemic of 1989-1990. Popul Trends $1991 ; 65: 16-21$.

2 Barker WH, Mulloolly JP. Influenza vaccination of elderly persons: reduction in pneumonia and influenza hospitalisations and deaths. FAMA 1980;244:2547-9.

3 British Thoracic Society. Guidelines for the management of community acquired pneumonia in adults admitted to hospital. Br f Hosp Med 1993;49:346-50.

4 Department of Health. Immunisation against infectious disease. London: HMSO, 1996: 167-72.

5 Butler JC, Breiman RF, Campbell JF, et al. Pneumococcal polysaccharide vaccine efficacy. An evaluation of current recommendations. FAMA 1993;270:1826-31.

6 Hirschman JV, Lipsky BA. The pneumococcal vaccine after 15 years of use. Arch Intern Med 1994;154:373-7.

7 Fiebach N, Beckett W. Prevention of respiratory infection in adults. Influenza and pneumococcal vaccines. Arch Intern Med 1994;154:2545-57.

8 McDonald P, Friedman EHI, Banks A, et al. Pneumococcal vaccine campaign based in general practice. BMF 1997;314: 1094 . 